QUARTERLY OF APPLIED MATHEMATICS

VOLUME LXVIII, NUMBER 1

MARCH 2010, PAGES 169-178

S 0033-569X(09)01151-3

Article electronically published on October 28, 2009

\title{
ENERGY SPLITTING FOR SOLUTIONS OF MULTI-DIMENSIONAL ISOTROPIC SYMMETRIC HYPERBOLIC SYSTEMS
}

\author{
BY \\ THOMAS C. SIDERIS \\ Department of Mathematics, University of California, Santa Barbara, CA 93106
}

1. Introduction. This note, submitted in honor of Walter Strauss' 70th birthday, offers a new look at the elegant paper by Costa and Strauss entitled Energy Splitting, 3. In it, they studied finite energy solutions to linear, constant coefficient, isotropic, symmetric hyperbolic systems. They showed that solutions separate, in $L^{2}$, into a superposition of outgoing plane waves, using a decomposition based on the Radon transform.

Our approach to energy splitting is based on the local energy decay result obtained by the author with B. Thomases in [9, for rotationally and scaling invariant isotropic systems. Inspired by Costa and Strauss, here we replace rotational invariance by the isospectral condition of $[3$. This involves a nonlocal modification of the rotational vector fields. However, the final result is entirely local. Solutions are decomposed locally according to the spectral projections of the symbol, and individual wave families concentrate near characteristic cones. After a few preliminaries, we state the main result and compare it with 3 . The proof is entirely self-contained. To conclude, we illustrate with three examples.

Energy splitting is closely related to the phenomenon of energy equipartition. In the context of symmetric hyperbolic systems with constant coefficients, there have been numerous works on these topics, among them [2, 4, 10, 11].

2. Preliminaries. Let $\mathcal{V}$ and $\mathcal{W}$ be finite-dimensional inner product spaces over $\mathbb{R}$. We will be concerned with $\mathcal{V}$-valued solutions $u:[0, T) \times \mathbb{R}^{n} \rightarrow \mathcal{V}$ (with $n>1$ ) of the linear system

$$
L(\partial) u=0 \quad \text { with } \quad L(\partial)=\partial_{t}+A(\nabla), \quad A(\nabla)=A_{k} \partial_{k},
$$

together with a system of constraints

$$
B(\nabla) u=0 \quad \text { with } \quad B(\nabla)=B_{k} \partial_{k} .
$$

Received October 8, 2008.

2000 Mathematics Subject Classification. Primary 35L45.

This research was partially sponsored by the National Science Foundation.

(C)2009 Brown University Reverts to public domain 28 years from publication 
Here, we suppose that the coefficients are constant linear maps

$$
A_{k} \in \mathcal{L}(\mathcal{V}, \mathcal{V}), \quad B_{k} \in \mathcal{L}(\mathcal{V}, \mathcal{W}), \quad k=1, \ldots, n
$$

Associated to the differential operators $A(\nabla)$ and $B(\nabla)$, define the symbols

$$
A(\xi)=A_{k} \xi^{k} \quad \text { and } \quad B(\xi)=B_{k} \xi^{k}, \quad \xi \in \mathbb{R}^{n} .
$$

We shall make three assumptions: a symmetry condition

$$
A(\xi)=A(\xi)^{*}, \quad \text { for all } \xi \in \mathbb{R}^{n},
$$

a nondegeneracy condition

$$
\operatorname{ker} B(\xi)=\operatorname{ker} A(\xi)^{\perp}, \quad \text { for all } \xi \in \mathbb{R}^{n},
$$

and an isospectral condition

$$
\text { The spectrum of } A(\omega) \text { is independent of } \omega \in S^{n-1} \text {. }
$$

The symmetry condition (A1) implies the conservation of energy. The nondegeneracy condition (A2) rules out stationary solutions. It is slightly stronger than the assumption $\operatorname{ker} A(\xi) \cap \operatorname{ker} B(\xi)=\{0\}$ made in 9 , but (A2) holds in all of the main examples. Systems satisfying the isospectral condition (A3) were called isotropic by Costa and Strauss because the wave speeds are independent of the direction of propagation. Condition (A3) is weaker than the rotational invariance condition assumed in [9], but the author is unaware of a physical system which satisfies (A3) and is not also rotationally invariant. In any case, (A3) is enough to construct a useful family of operators which generalize the usual rotational vector fields.

Before coming to the main result, we mention a few simple facts.

Spectral projections. Let $\omega \in S^{n-1}$. Using $\sigma$ to denote the spectrum, we have by (A3) that $\sigma(A(\omega))=\sigma(A(-\omega))=\sigma(-A(\omega))$; it follows that the nonzero eigenvalues of $A(\omega)$ occur in plus/minus pairs. Denote the distinct eigenvalues of $A(\omega)$ by $\left\{\lambda_{\alpha}: \alpha=\right.$ $-m, \ldots, m\}$, labeled so that

$$
\lambda_{0}=0<\lambda_{1}<\ldots<\lambda_{m}, \quad \text { and } \quad \lambda_{-\alpha}=-\lambda_{\alpha} .
$$

For each $\omega \in S^{n-1}$, the orthogonal projection of $\mathcal{V}$ onto the eigenspace of $A(\omega)$ corresponding to the eigenvalue $\lambda_{\beta}$ is given by

$$
\mathcal{P}_{\beta}(\omega)=\prod_{\alpha \neq \beta} \frac{A(\omega)-\lambda_{\alpha} I}{\lambda_{\beta}-\lambda_{\alpha}} .
$$

The orthogonal projections $\mathcal{P}_{\beta}(\omega)$ are smooth functions of $\omega$ on $S^{n-1}$.

\section{Main result.}

Theorem 1. Suppose that $u$ solves (1a), (1b) with $n>1$. Assume that (A1), (A2), (A3) hold. Select $\delta$ so that

$$
0<\delta<\min \left\{\left|\lambda_{\alpha}-\lambda_{\beta}\right| / 2: \alpha \neq \beta\right\} .
$$


If the initial data satisfies $u_{0} \in L^{2}\left(\mathbb{R}^{n}\right)$, then

$$
\left\|u(t)-\sum_{\alpha=1}^{m} \chi_{\left\{\left|\lambda_{\alpha} t-r\right|<\delta t\right\}} \mathcal{P}_{\alpha}(x / r) u(t)\right\|_{L^{2}\left(\mathbb{R}^{n}\right)} \rightarrow 0,
$$

as $t \rightarrow \infty$. (Here, $\chi_{\Omega}$ denotes the characteristic function of a set $\Omega$.)

If $u_{0} \in L^{2}\left(\mathbb{R}^{n}\right)$ and $(1+|x|)\left|\nabla u_{0}\right| \in L^{2}\left(\mathbb{R}^{n}\right)$, then for $k=1, \ldots, n$,

$$
\left\|\partial_{k} u(t)-\sum_{\alpha=1}^{m} \chi_{\left\{\left|\lambda_{\alpha} t-r\right|<\delta t\right\}} \mathcal{P}_{\alpha}(x / r) \partial_{k} u(t)\right\|_{L^{2}\left(\mathbb{R}^{n}\right)}=\mathcal{O}\left(t^{-1}\right),
$$

as $t \rightarrow \infty$.

This result has the advantage of using only local quantities. Note, in particular, that the projections act in the physical variables and not the phase variables and that the characteristic functions separate the wave families and localize them near their light cones. The asymptotic analysis of certain nonlinear quantities in the solution (such as null forms) therefore becomes potentially quite simple.

Equation (3a) implies that

$$
\mathcal{P}_{\alpha}(x / r) u(t, x) \rightarrow 0 \quad \text { in } L^{2}\left(\mathbb{R}^{n}\right) \text { as } t \rightarrow \infty, \quad \alpha=-m, \ldots, 0 .
$$

On the other hand, the result of Costa and Strauss [3] says that

$$
\left(\lambda_{\alpha} I-A(x / r)\right) u^{(\alpha)}(t) \rightarrow 0 \text { in } L^{2}\left(\mathbb{R}^{n}\right) \text { as } t \rightarrow \infty, \alpha=1, \ldots, m,
$$

where $u^{(\alpha)}$ is a nonlocal plane wave decomposition involving waves with propagation speeds $\pm \lambda_{\alpha}$. If we make the asymptotically correct identification $u^{(\alpha)}(t, x) \sim\left(\mathcal{P}_{\alpha}(x / r)+\right.$ $\left.\mathcal{P}_{-\alpha}(x / r)\right) u(t, x)$, then (4b) and (4a) are seen to be equivalent.

4. Commutation properties. Define the usual angular momentum and scaling operators

$$
\Omega_{i j}=x^{i} \partial_{j}-x^{j} \partial_{i} \quad \text { and } \quad S=t \partial_{t}+r \partial_{r} .
$$

The angular momentum operators must be "corrected" in order that they commute with $A(\nabla)$ and $B(\nabla)$. For rotationally invariant systems, one uses the natural Lie derivatives, resulting in a constant linear map as a correction term; see [9], for example. Here, we must generalize this construction slightly.

The operators $\Omega_{i j}$ are invariant under Fourier transform, that is, $\Omega_{i j}=\mathcal{F}^{-1} \Omega_{i j} \mathcal{F}$. Define the family of operators

$$
\widetilde{\Omega}_{i j}=\sum_{\alpha=-m}^{m} \mathcal{F}^{-1} \mathcal{P}_{\alpha}(\xi /|\xi|) \Omega_{i j} \mathcal{P}_{\alpha}(\xi /|\xi|) \mathcal{F},
$$

with $\xi \in \mathbb{R}^{n}$ being the Fourier transform variable.

Lemma 1. If $u, \Omega_{i j} u \in L^{2}\left(\mathbb{R}^{n}\right)$, then $\widetilde{\Omega}_{i j} u \in L^{2}\left(\mathbb{R}^{n}\right)$, and

$$
\left\|\widetilde{\Omega}_{i j} u-\Omega_{i j} u\right\|_{L^{2}\left(\mathbb{R}^{n}\right)} \leq C\|u\|_{L^{2}\left(\mathbb{R}^{n}\right)} .
$$


Proof. From the definition of $\widetilde{\Omega}_{i j}$, we see that

$$
\widetilde{\Omega}_{i j}=I \Omega_{i j}+Z_{i j}(\nabla),
$$

where the correction term $Z_{i j}(\nabla)$ is an operator whose symbol is homogeneous of degree zero and is therefore bounded in $L^{2}$.

Lemma 2. Let $u(t)$ be a solution of (1a) with initial data $u_{0}$ satisfying

$$
u_{0} \in L^{2}\left(\mathbb{R}^{n}\right) \quad \text { and } \quad(1+|x|)\left|\nabla u_{0}\right| \in L^{2}\left(\mathbb{R}^{n}\right) .
$$

Then the functions $u(t), \widetilde{\Omega}_{i j} u(t), S u(t)$ lie in $L^{2}\left(\mathbb{R}^{n}\right)$ for all $t \in \mathbb{R}$, they solve (1a), and their $L^{2}$-norms are conserved.

Proof. The assumptions on the initial data imply that $u_{0}, r \partial_{r} u_{0}$, and $\Omega_{i j} u_{0}$ lie in $L^{2}\left(\mathbb{R}^{n}\right)$. By Lemma 1, $\widetilde{\Omega}_{i j} u_{0}$ also lies in $L^{2}\left(\mathbb{R}^{n}\right)$.

The solution of (1a) is represented by $u(t)=T(t) u_{0}=\mathcal{F}^{-1} \exp i t A(\xi) \mathcal{F} u_{0}$. By the symmetry condition (A1), the solution operator $T(t)$ is an isometry on $L^{2}\left(\mathbb{R}^{n}\right)$. Thus, $\|u(t)\|_{L^{2}\left(\mathbb{R}^{n}\right)}=\left\|u_{0}\right\|_{L^{2}\left(\mathbb{R}^{n}\right)}$.

Since $\Omega_{i j}|\xi|=0$, we have from the definition of $\widetilde{\Omega}_{i j}$ that $\left[\widetilde{\Omega}_{i j}, A(\nabla)\right]=0$, and hence $\left[\widetilde{\Omega}_{i j}, L(\partial)\right]=0$. It follows that $\widetilde{\Omega}_{i j} u(t)=T(t) \widetilde{\Omega}_{i j} u_{0}$ solves (19a), and $\left\|\widetilde{\Omega}_{i j} u(t)\right\|_{L^{2}\left(\mathbb{R}^{n}\right)}=$ $\left\|\widetilde{\Omega}_{i j} u_{0}\right\|_{L^{2}\left(\mathbb{R}^{n}\right)}$. Likewise, since $[S, L(\partial)]=-L(\partial)$, we have that $S u(t)=T(t)\left[r \partial_{r} u_{0}\right]$, with conservation of the norm.

5. Proof of Theorem 1. The proof of Theorem 11depends on the following version of the result in [9] specialized to the homogeneous, inviscid case. We provide its short proof for completeness.

Theorem 2. Assume that (A1), (A2), (A3) hold. Let $u$ be a solution of (1a), (1b) with $n>1$ whose initial data satisfies

$$
u_{0} \in L^{2}\left(\mathbb{R}^{n}\right) \quad \text { and } \quad(1+|x|)\left|\nabla u_{0}\right| \in L^{2}\left(\mathbb{R}^{n}\right) .
$$

Then there are positive constants $\mu$ and $C$, depending on the coefficients $A_{k}$ and $B_{k}$, such that the first derivatives $\partial_{k} u, k=1, \ldots, n$ satisfy the estimates

$$
\mu t\left\|\partial_{k} u(t)\right\|_{L^{2}(\{r \leq \mu t\})} \leq C\left\|u_{0}\right\|_{L^{2}\left(\mathbb{R}^{n}\right)}+\left\|r \partial_{r} u_{0}\right\|_{L^{2}\left(\mathbb{R}^{n}\right)},
$$

and for any $\nu>0$,

$$
\begin{aligned}
\left\|\left(\lambda_{\alpha} t-r\right) \mathcal{P}_{\alpha}(x /|x|) \partial_{k} u(t)\right\|_{L^{2}(\{r \geq \nu t\})} & \\
& \leq C\left[\left\|\widetilde{\Omega} u_{0}\right\|_{L^{2}\left(\mathbb{R}^{n}\right)}+\left\|u_{0}\right\|_{L^{2}\left(\mathbb{R}^{n}\right)}\right]+\left\|r \partial_{r} u_{0}\right\|_{L^{2}\left(\mathbb{R}^{n}\right)},
\end{aligned}
$$

for each $\alpha=-m, \ldots, m$.

Proof. By (A2), the expression $|A(\omega) u|_{\mathcal{V}}^{2}+|B(\omega) u|_{\mathcal{W}}^{2}$ vanishes if and only if $u=0$. In other words, the map $A(\omega)^{2}+B(\omega)^{*} B(\omega)$ in $\mathcal{L}(\mathcal{V}, \mathcal{V})$ is positive definite for each $\omega \in S^{n-1}$, and so there exists a constant $\mu$ such that

$$
(3 \mu)^{2}|u|_{\mathcal{V}}^{2} \leq|A(\omega) u|_{\mathcal{V}}^{2}+|B(\omega) u|_{\mathcal{W}}^{2},
$$


for all $u \in \mathcal{V}$ and $\omega \in S^{n-1}$. Therefore, using the Fourier transform, we obtain

$$
3 \mu\|\nabla u\|_{L^{2}\left(\mathbb{R}^{n}\right)} \leq\|A(\nabla) u\|_{L^{2}\left(\mathbb{R}^{n}\right)}+\|B(\nabla) u\|_{L^{2}\left(\mathbb{R}^{n}\right)},
$$

for all functions $u \in H^{1}\left(\mathbb{R}^{n}\right)$.

Introduce a cutoff function $\zeta \in C^{\infty}(\mathbb{R})$ with $0 \leq \zeta \leq 1$ and

$$
\zeta(s)= \begin{cases}1, & \text { if } s \leq 1 \\ 0, & \text { if } s \geq 2\end{cases}
$$

Fixing $\mu$ as in (6), define $\psi_{\mu}(t, r)=\zeta(r /(\mu t))$.

Let $u$ solve (1a), (1b), and set $v=\psi_{\mu} u$, so that $v$ is supported in $\{r \leq 2 \mu t\}$. By (6), we obtain

$$
\begin{aligned}
3 \mu t\|\nabla v\|_{L^{2}\left(\mathbb{R}^{n}\right)} \leq t\|A(\nabla) v\|_{L^{2}\left(\mathbb{R}^{n}\right)}+t\|B(\nabla) v\|_{L^{2}\left(\mathbb{R}^{n}\right)} & \\
& \leq\left\|\left(t A(\nabla)-r \partial_{r}\right) v\right\|_{L^{2}\left(\mathbb{R}^{n}\right)}+2 \mu t\|\nabla v\|_{L^{2}\left(\mathbb{R}^{n}\right)}+t\|B(\nabla) v\|_{L^{2}\left(\mathbb{R}^{n}\right)} .
\end{aligned}
$$

This yields the bound

$$
\mu t\|\nabla v\|_{L^{2}\left(\mathbb{R}^{n}\right)} \leq\left\|\left(t A(\nabla)-r \partial_{r}\right) v\right\|_{L^{2}\left(\mathbb{R}^{n}\right)}+t\|B(\nabla) v\|_{L^{2}\left(\mathbb{R}^{n}\right)} .
$$

Now since $(\mu t+r)\left|\partial_{k} \psi_{\mu}\right| \leq C$, we have from (8) the estimate

$$
\begin{aligned}
\mu t\left\|\partial_{k} u\right\|_{L^{2}(\{r \leq \mu t\})} & \\
& =\mu t\left\|\partial_{k} v\right\|_{L^{2}(\{r \leq \mu t\})} \\
& \leq \mu t\left\|\partial_{k} v\right\|_{L^{2}\left(\mathbb{R}^{n}\right)} \\
& \leq\left\|\left(t A(\nabla)-r \partial_{r}\right) v\right\|_{L^{2}\left(\mathbb{R}^{n}\right)}+t\|B(\nabla) v\|_{L^{2}\left(\mathbb{R}^{n}\right)} \\
& \leq\left\|\left(t A(\nabla)-r \partial_{r}\right) u\right\|_{L^{2}\left(\mathbb{R}^{n}\right)}+t\|B(\nabla) u\|_{L^{2}\left(\mathbb{R}^{n}\right)}+C\|u\|_{L^{2}\left(\mathbb{R}^{n}\right)} \\
& =\|t L(\partial) u-S u\|_{L^{2}\left(\mathbb{R}^{n}\right)}+t\|B(\nabla) u\|_{L^{2}\left(\mathbb{R}^{n}\right)}+C\|u\|_{L^{2}\left(\mathbb{R}^{n}\right)} .
\end{aligned}
$$

But since $u$ solves (1a), (1b), this equals

$$
\|S u\|_{L^{2}\left(\mathbb{R}^{n}\right)}+C\|u\|_{L^{2}\left(\mathbb{R}^{n}\right)} .
$$

The estimate (5a) now follows from Lemma 2 , 
Using the orthogonal projections $\mathcal{P}_{\alpha}(\omega)$, with $\omega=x / r$, and the vector fields $\Omega_{i j}$, we have the pointwise estimate

$$
\begin{aligned}
\left|\left(\lambda_{\alpha} t-r\right) \mathcal{P}_{\alpha}(\omega) \partial_{j} u(t, x)\right|_{\mathcal{v}} \\
=\left|\mathcal{P}_{\alpha}(\omega)(t A(\omega)-r I) \partial_{j} u(t, x)\right|_{\mathcal{v}} \\
\leq\left|(t A(\omega)-r I) \partial_{j} u(t, x)\right|_{\mathcal{v}} \\
=\left|\left(t A_{k}-r \omega^{k} I\right) \omega^{k} \partial_{j} u(t, x)\right|_{\mathcal{v}} \\
=\left|\left(t A_{k}-r \omega^{k} I\right)\left(\omega^{j} \partial_{k}+\frac{1}{r} \Omega_{k j}\right) u(t, x)\right|_{\mathcal{v}} \\
=\left|\left[\omega^{j}\left(t A(\nabla)-r \partial_{r}\right)+\left(t A_{k}-r \omega^{k} I\right) \frac{1}{r} \Omega_{k j}\right] u(t, x)\right|_{\mathcal{v}} \\
\leq\left|\left(t A(\nabla)-r \partial_{r}\right) u(t, x)\right|_{\mathcal{v}}+C\left|\frac{t}{r}+1\right||\Omega u(t, x)|_{\mathcal{v}} \\
=|S u(t, x)|_{\mathcal{v}}+C\left|\frac{t}{r}+1\right||\Omega u(t, x)|_{\mathcal{v}} .
\end{aligned}
$$

An integration over the region $\{r \geq \nu t\}$ yields

$$
\left\|\left(\lambda_{\alpha} t-r\right) \mathcal{P}_{\alpha}(x /|x|) \partial_{j} u(t)\right\|_{L^{2}(\{r \geq \nu t\})} \leq\|S u(t)\|_{L^{2}\left(\mathbb{R}^{n}\right)}+C\|\Omega u(t)\|_{L^{2}\left(\mathbb{R}^{n}\right)} .
$$

The estimate (5b) now follows by an application of Lemmas 1 and 2

As a final preparation, we need the following approximation result.

Lemma 3. If $u \in L^{2}\left(\mathbb{R}^{n}\right)$ satisfies the constraint equation (1b), then for any $\varepsilon>0$, $k=1, \ldots, n$, there exists $v^{\varepsilon} \in \mathcal{S}\left(\mathbb{R}^{n}\right)$ satisfying (1b), as well as the estimate

$$
\left\|u-\partial_{k} v^{\varepsilon}\right\|_{L^{2}\left(\mathbb{R}^{n}\right)}<\varepsilon .
$$

Proof. A function $u \in L^{2}\left(\mathbb{R}^{n}\right)$ satisfies the constraint equation (1b) if and only if $\mathcal{F} u(\xi) \in \operatorname{ker} B(\eta), \eta=\xi /|\xi|$. By (ㅈ2), $\operatorname{ker} A(\eta)^{\perp}=\operatorname{ker} B(\eta)$, so this is equivalent to saying

$$
\mathcal{F} u(\xi)=Q(\eta) \mathcal{F} u(\xi), \quad Q(\eta)=I-\mathcal{P}_{0}(\eta),
$$

where $\mathcal{P}_{0}(\eta)$ is the orthogonal projection of $\mathcal{V}$ onto $\operatorname{ker} A(\eta)$.

The class $\mathcal{S}\left(\mathbb{R}^{n}\right)$ is dense in $L^{2}\left(\mathbb{R}^{n}\right)$, so we may choose $\mathcal{F} w \in \mathcal{S}\left(\mathbb{R}^{n}\right)$, such that $\|\mathcal{F} u-\mathcal{F} w\|_{L^{2}\left(\mathbb{R}^{n}\right)}<\varepsilon / 2$. Fix $k=1, \ldots, n$, and, using the cutoff function (7), choose $\delta=\delta(\varepsilon)>0$ small enough so that

$$
\left\|\zeta\left(\xi^{k} / \delta\right) \mathcal{F} w\right\|_{L^{2}\left(\mathbb{R}^{n}\right)}<\varepsilon / 2 .
$$

Finally, define $\mathcal{F} v^{\varepsilon}=\left(i \xi^{k}\right)^{-1} Q(\eta)\left(1-\zeta\left(\xi^{k} / \delta\right)\right) \mathcal{F} w(\xi)$. Then $v^{\varepsilon} \in \mathcal{S}\left(\mathbb{R}^{n}\right), v^{\varepsilon}$ satisfies (1b), and we have the estimate

$$
\begin{aligned}
\left\|u-\partial_{k} v^{\varepsilon}\right\|_{L^{2}\left(\mathbb{R}^{n}\right)} & =\left\|\mathcal{F} u(\xi)-Q(\eta)\left(1-\zeta\left(\xi^{k} / \delta\right)\right) \mathcal{F} w(\xi)\right\|_{L^{2}\left(\mathbb{R}^{n}\right)} \\
& =\left\|Q(\eta)\left(\mathcal{F} u(\xi)-\left(1-\zeta\left(\xi^{k} / \delta\right)\right) \mathcal{F} w(\xi)\right)\right\|_{L^{2}\left(\mathbb{R}^{n}\right)} \\
& \leq\left\|\mathcal{F} u(\xi)-\left(1-\zeta\left(\xi^{k} / \delta\right)\right) \mathcal{F} w(\xi)\right\|_{L^{2}\left(\mathbb{R}^{n}\right)} \\
& \leq\|\mathcal{F} u-\mathcal{F} w\|_{L^{2}\left(\mathbb{R}^{n}\right)}+\left\|\zeta\left(\xi^{k} / \delta\right) \mathcal{F} w\right\|_{L^{2}\left(\mathbb{R}^{n}\right)} \\
& <\varepsilon / 2+\varepsilon / 2=\varepsilon .
\end{aligned}
$$


Proof of Theorem 1, (3b). Choose $0<\delta<\min \left\{\left|\lambda_{\alpha}-\lambda_{\beta}\right| / 2: \alpha \neq \beta\right\}$. Assume that $u_{0}$ satisfies the assumptions of Theorem 2 .

If $\alpha=-m, \ldots, 0$, then $\lambda_{\alpha} \leq 0$, and so for $r>\nu t$, we have $\left|\lambda_{\alpha} t-r\right| \geq \nu t$. Taking $\nu=\mu$ in Theorem 2 and combining (5a), (5b), we see that

$$
\mu t\left\|\mathcal{P}_{\alpha}(x /|x|) \partial_{k} u(t)\right\|_{L^{2}\left(\mathbb{R}^{n}\right)} \leq C,
$$

for $\alpha=-m, \ldots, 0$.

On the other hand, if $\alpha=1, \ldots, m$, we write

$$
\begin{aligned}
\delta t \chi_{\left\{\left|\lambda_{\alpha} t-r\right|>\delta t\right\}} & =\delta t \chi_{\left\{\left|\lambda_{\alpha} t-r\right|>\delta t\right\} \cap\{r<\delta t\}}+\delta t \chi_{\left\{\left|\lambda_{\alpha} t-r\right|>\delta t\right\} \cap\{r>\delta t\}} \\
& \leq \delta t \chi_{\{r<\delta t\}}+\left|\lambda_{\alpha} t-r\right| \chi_{\{r>\delta t\}} .
\end{aligned}
$$

Thus, taking $\nu=\delta$ in (5b), we get from Theorem 2 ,

$$
\begin{aligned}
& \delta t\left\|\mathcal{P}_{\alpha}(x /|x|) \partial_{k} u(t)\right\|_{L^{2}\left(\left\{\left|\lambda_{\alpha} t-r\right|>\delta t\right)\right\}} \\
& \quad \leq \delta t\left\|\mathcal{P}_{\alpha}(x /|x|) \partial_{k} u(t)\right\|_{L^{2}(\{r<\delta t)\}} \\
& \quad+\left\|\left(\lambda_{\alpha} t-r\right) \mathcal{P}_{\alpha}(x /|x|) \partial_{k} u(t)\right\|_{L^{2}(\{r>\delta t\})}
\end{aligned}
$$

$$
\leq C \text {. }
$$

We conclude that

$$
\begin{aligned}
& \left\|\partial_{k} u(t)-\sum_{\alpha=1}^{m} \chi_{\left\{\left|\lambda_{\alpha} t-r\right|<\delta t\right\}} \mathcal{P}_{\alpha}(x /|x|) \partial_{k} u(t)\right\|_{L^{2}\left(\mathbb{R}^{n}\right)} \\
& \leq \sum_{\alpha=-m}^{0}\left\|\mathcal{P}_{\alpha}(x /|x|) \partial_{k} u(t)\right\|_{L^{2}\left(\mathbb{R}^{n}\right)} \\
& \quad+\sum_{\alpha=1}^{m}\left\|\mathcal{P}_{\alpha}(x /|x|) \partial_{k} u(t)\right\|_{L^{2}\left(\left\{\left|\lambda_{\alpha} t-r\right|>\delta t\right\}\right)} \\
& \leq C t^{-1}
\end{aligned}
$$

thereby proving (3b).

Proof of Theorem 1, (3a). Now assume merely that $u_{0} \in L^{2}\left(\mathbb{R}^{n}\right)$. Let $\varepsilon^{\prime}>0$ be given. Using Lemma 3 with $\varepsilon=\varepsilon^{\prime} / 3$, choose $v_{0}^{\varepsilon} \in \mathcal{S}\left(\mathbb{R}^{n}\right)$ satisfying (1b) and

$$
\left\|u_{0}-\partial_{k} v_{0}^{\varepsilon}\right\|_{L^{2}\left(\mathbb{R}^{n}\right)}<\varepsilon,
$$

for some $k=1, \ldots, n$.

Let $v^{\varepsilon}$ solve (1a), (1b) with initial data $v_{0}^{\varepsilon}$. By energy conservation, we have that

$$
\left\|u(t)-\partial_{k} v^{\varepsilon}(t)\right\|_{L^{2}\left(\mathbb{R}^{n}\right)}=\left\|u_{0}-\partial_{k} v_{0}^{\varepsilon}\right\|_{L^{2}\left(\mathbb{R}^{n}\right)}<\varepsilon .
$$

Moreover, we have that

$$
\left\|\sum_{\alpha=1}^{m} \chi_{\left\{\left|\lambda_{\alpha} t-r\right|<\delta\right\}} \mathcal{P}_{\alpha}(x /|x|)\left(u(t)-\partial_{k} v^{\varepsilon}(t)\right)\right\|_{L^{2}\left(\mathbb{R}^{n}\right)} \leq\left\|u(t)-\partial_{k} v^{\varepsilon}(t)\right\|_{L^{2}\left(\mathbb{R}^{n}\right)}<\varepsilon .
$$


Adding and subtracting $\partial_{k} v^{\varepsilon}$, we estimate as follows:

$$
\begin{aligned}
\| u(t)- & \sum_{\alpha=1}^{m} \chi_{\left\{\left|\lambda_{\alpha} t-r\right|<\delta t\right\}} \mathcal{P}_{\alpha}(x /|x|) u(t) \|_{L^{2}\left(\mathbb{R}^{n}\right)} \\
\leq & \left\|u(t)-\partial_{k} v^{\varepsilon}(t)\right\|_{L^{2}\left(\mathbb{R}^{n}\right)} \\
& +\left\|\partial_{k} v^{\varepsilon}(t)-\sum_{\alpha=1}^{m} \chi_{\left\{\left|\lambda_{\alpha} t-r\right|<\delta t\right\}} \mathcal{P}_{\alpha}(x /|x|) \partial_{k} v^{\varepsilon}(t)\right\|_{L^{2}\left(\mathbb{R}^{n}\right)} \\
& +\left\|\sum_{\alpha=1}^{m} \chi_{\left\{\left|\lambda_{\alpha} t-r\right|<\delta t\right\}} \mathcal{P}_{\alpha}(x /|x|)\left(u(t)-\partial_{k} v^{\varepsilon}(t)\right)\right\|_{L^{2}\left(\mathbb{R}^{n}\right)}
\end{aligned}
$$

The first and third terms are both smaller than $\varepsilon=\varepsilon^{\prime} / 3$, while the middle term is $\mathcal{O}\left(t^{-1}\right)$. Therefore, for $t$ sufficiently large, the sum of the three terms is smaller than $\varepsilon^{\prime}$.

\section{Examples.}

Wave equation. The wave equation for $\phi: \mathbb{R} \times \mathbb{R}^{n} \rightarrow \mathbb{R}$,

$$
\partial_{t}^{2} \phi-c^{2} \Delta \phi=0,
$$

can be written in the form (1a) using $u=\partial \phi=\left(\partial_{t} \phi, \nabla \phi\right) \in \mathcal{V}=\mathbb{R}^{n+1}$ with the inner product $\operatorname{diag}\left(c^{-2}, 1, \ldots, 1\right)$. The coefficient matrices are

$$
-A_{k}=c^{2} e_{0} \otimes e_{k}+e_{k} \otimes e_{0}, \quad k=1, \ldots, n,
$$

where $\left\{e_{a}\right\}_{a=0}^{n}$ denotes the standard basis on $\mathcal{V}$. The constraint equations (1b) consist of

$$
\partial_{i} u^{j}-\partial_{j} u^{i}=0, \quad i, j=1, \ldots, n .
$$

The assumptions (A1)-(A3) are easily verified; see [9].

The symbol has eigenvalues $0, \pm c$, and from (2),

$$
\mathcal{P}_{1}(x / r)=\left(2 c^{2}\right)^{-1}\left[A(x / r)^{2}+A(x / r)\right] .
$$

After a bit of calculation, Theorem 1 gives

$$
\left[\begin{array}{c}
\partial_{t} \phi(t) \\
\nabla \phi(t)
\end{array}\right]-\chi_{\{|c t-r|<c t / 2\}} \frac{1}{2}\left[\begin{array}{c}
\partial_{t} \phi(t)-c \partial_{r} \phi(t) \\
\left(\partial_{r} \phi(t)-c^{-1} \partial_{t} \phi(t)\right)(x / r)
\end{array}\right] \rightarrow 0,
$$

in $L^{2}\left(\mathbb{R}^{n}\right)$, as $t \rightarrow \infty$.

Maxwell's equation. The homogeneous Maxwell system for an electromagnetic field $(E, H): \mathbb{R} \times \mathbb{R}^{3} \rightarrow \mathbb{R}^{3} \times \mathbb{R}^{3}$ takes the form

$$
\begin{aligned}
& \varepsilon \partial_{t} E-c \nabla \wedge H=0, \\
& \mu \partial_{t} H+c \nabla \wedge E=0,
\end{aligned}
$$

with the constraints

$$
\nabla \cdot \varepsilon E=0, \quad \nabla \cdot \mu H=0 .
$$

Taking $\mathcal{V}=\mathbb{R}^{3} \times \mathbb{R}^{3}$ with the inner product

$$
\left\langle u, u^{\prime}\right\rangle_{\mathcal{\nu}}=\left\langle(E, H),\left(E^{\prime}, H^{\prime}\right)\right\rangle_{\mathcal{\nu}}=\varepsilon\left\langle E, E^{\prime}\right\rangle_{\mathbb{R}^{3}}+\mu\left\langle H, H^{\prime}\right\rangle_{\mathbb{R}^{3}},
$$

the system satisfies the assumptions of Theorem 1 . 
In this case, the symbol is given by

$$
A\left(\frac{x}{r}\right) u=\left(-\frac{c}{\varepsilon} \frac{x}{r} \wedge H, \frac{c}{\mu} \frac{x}{r} \wedge E\right),
$$

and the eigenvalues are $0, \pm \lambda= \pm c / \sqrt{\varepsilon \mu}$.

Once again, using (2), we have

$$
\mathcal{P}_{1}(x / r)=\frac{1}{2 \lambda^{2}}\left(A(x / r)^{2}+A(x / r)\right) .
$$

Unravelling Theorem 1, we find that

$$
\left[\begin{array}{l}
E \\
H
\end{array}\right]-\chi_{\left\{|\lambda t-r|<\frac{\lambda t}{2}\right\}} \frac{1}{2}\left[\begin{array}{l}
E-\langle E, x / r\rangle_{\mathbb{R}^{3}}(x / r)-\frac{\mu}{c}(x / r) \wedge H \\
H-\langle H, x / r\rangle_{\mathbb{R}^{3}}(x / r)+\frac{\varepsilon}{c}(x / r) \wedge E
\end{array}\right] \rightarrow 0
$$

in $L^{2}\left(\mathbb{R}^{3}\right)$, as $t \rightarrow \infty$. See also [1, 5, 8].

Linear isotropic elasticity. In linear isotropic elasticity, the material displacement vector $\phi: \mathbb{R} \times \mathbb{R}^{3} \rightarrow \mathbb{R}^{3}$ satisfies the system

$$
\partial_{t}^{2} \phi-c_{2}^{2} \Delta \phi-\left(c_{1}^{2}-c_{2}^{2}\right) \nabla(\nabla \cdot \phi)=0, \quad c_{1}>c_{2}>0 .
$$

This can be written in the form (1a) using the deformation gradient $F_{\ell}^{i}=\partial_{\ell} \phi^{i}$ and velocity $\partial_{t} \phi^{i}$ as variables:

$$
\begin{aligned}
& \partial_{t} F-\nabla v=0, \\
& \partial_{t} v-\nabla \cdot T F=0,
\end{aligned}
$$

in which $T \in \mathcal{L}\left(\mathbb{R}^{3} \otimes \mathbb{R}^{3}, \mathbb{R}^{3} \otimes \mathbb{R}^{3}\right)$ is defined by

$$
T F=c_{2}^{2} F+\left(c_{1}^{2}-c_{2}^{2}\right) \operatorname{tr} F I .
$$

The constraints (1b) are

$$
\partial_{m} F_{\ell}^{i}-\partial_{\ell} F_{m}^{i}=0, \quad i, \ell, m=1,2,3 .
$$

The natural vector space is therefore $\mathcal{V}=\left(\mathbb{R}^{3} \otimes \mathbb{R}^{3}\right) \times \mathbb{R}^{3}$, and with the inner product

$$
\langle u, \bar{u}\rangle_{\mathcal{v}}=\langle(F, v),(\bar{F}, \bar{v})\rangle_{\mathcal{v}}=\operatorname{tr}\left[(T F) \bar{F}^{*}\right]+\langle v, \bar{v}\rangle_{\mathbb{R}^{3}},
$$

the system (9a)-(9d) satisfies the conditions of Theorem 1

The symbol $A(\omega) \in \mathcal{L}(\mathcal{V}, \mathcal{V})$ is given by

$$
A(\omega) u=A(\omega)(F, v)=-(v \otimes \omega, T F \omega),
$$

which has eigenvalues $0, \pm c_{2}, \pm c_{1}$. Thus, this system has multiple propagation speeds. The principal components, according to Theorem 1, are a one-dimensional family of fast pressure waves

$$
\begin{gathered}
\mathcal{P}_{f} u=\frac{1}{2 c_{1}^{2}}\left[c_{1}\left\langle w_{f}, \frac{x}{r}\right\rangle-\left(c_{1}^{2}-c_{2}^{2}\right)\left(\nabla \cdot \phi-\left\langle\partial_{r} \phi, \frac{x}{r}\right\rangle\right)\right] \cdot\left(-\frac{x}{r} \otimes \frac{x}{r}, c_{1} \frac{x}{r}\right), \\
w_{f}=\partial_{t} \phi-c_{1} \partial_{r} \phi .
\end{gathered}
$$

and a two-dimensional family of slow shear waves

$$
\begin{gathered}
\mathcal{P}_{s} u=\frac{1}{2 c_{2}^{2}}\left(-c_{2}\left(w_{s}-\left\langle w_{s}, \frac{x}{r}\right\rangle \frac{x}{r}\right) \otimes \frac{x}{r}, c_{2}^{2}\left(w_{s}-\left\langle w_{s}, \frac{x}{r}\right\rangle \frac{x}{r}\right)\right), \\
w_{s}=\partial_{t} \phi-c_{2} \partial_{r} \phi .
\end{gathered}
$$


With $\delta=\min \left\{c_{2} / 2,\left(c_{1}-c_{2}\right) / 2\right\}$, we have

$$
u-\chi_{\left\{\left|c_{1} t-r\right|<\delta t\right\}} \mathcal{P}_{f} u-\chi_{\left\{\left|c_{2} t-r\right|<\delta t\right\}} \mathcal{P}_{s} u \rightarrow 0, \quad \text { in } L^{2}\left(\mathbb{R}^{3}\right), \text { as } t \rightarrow \infty .
$$

See also [6, 7].

\section{REFERENCES}

[1] Alinhac, S. Remarks on energy inequalities for wave and Maxwell equations on a curved background. Math. Ann. 3294 (2004), 707-722. MR2076683 (2005f:58047)

[2] Bachelot, A. Équipartition de l'énergie pour les systèmes hyperboliques et formes compatibles. Ann. Inst. H. Poincaré Phys. Théor. 461 (1987), 45-76. MR877995 (88a:35144)

[3] Costa, D.G. and Strauss, W.A. Energy splitting. Quart. Appl. Math. 393 (1981/82), 351-361. MR636240 (83d:35090)

[4] Costa, D.G. On partition of energy for uniformly propagative systems. J. Math. Anal. Appl. 581 (1977), 56-62. MR0470505 (57:10256)

[5] Christodoulou, D. and Klainerman, S. Asymptotic properties of linear field equations in Minkowski space. Comm. Pure Appl. Math. 432 (1990), 137-199. MR1038141 (91a:58202)

[6] Dassios, G. and Galanis, E. Asymptotic equipartition of kinetic and strain energy for elastic waves in anisotropic media. Quart. Appl. Math. 381 (1980/81), 121-128. MR575835 (81e:73029)

[7] Dassios, G. and Grillakis, M. Equipartition of energy for anisotropic elastic waves. J. Differential Equations 513 (1984), 408-418. MR735206 (85e:73016)

[8] Glassey, R.T. and Strauss, W.A. Decay of classical Yang-Mills fields. Comm. Math. Phys. 651 (1979), 1-13. MR526974 (80c:81064)

[9] Sideris, T.C. and Thomases, B. Local energy decay for solutions of multi-dimensional isotropic symmetric hyperbolic systems. J. Hyperbolic Differ. Equ. 34 (2005), 673-690. MR2289610 (2007k:35306)

[10] Strichartz, R.S. Asymptotic behavior of waves. J. Funct. Anal. 403 (1981), 341-357. MR611588 (82j:35020)

[11] Wilcox, C. Asymptotic wave functions and energy distributions in strongly propagative anisotropic media. J. Math. Pures Appl. 573 (1978), 275-321. MR513101 (80j:35082) 